\title{
Microthrombotic renal involvement in an SLE patient with concomitant catastrophic antiphospholipid syndrome - The beneficial effect of Rituximab treatment
}

\begin{tabular}{|c|c|}
\hline Journal: & Lupus \\
\hline Manuscript ID & Draft \\
\hline Manuscript Type: & Case Report \\
\hline Date Submitted by the Author: & $\mathrm{n} / \mathrm{a}$ \\
\hline Complete List of Authors: & $\begin{array}{l}\text { Diószegi, Ágnes; University of Debrecen, Faculty of Medicine, University of } \\
\text { Debrecen, Faculty of Medicine, Department of Internal Medicine, Division of } \\
\text { Angiology } \\
\text { Tarr, Tunde; University of Debrecen, 3rd Dept. of Internal Medicine } \\
\text { Nagy-Vincze, Melinda; University of Debrecen, Faculty of Medicine, Clinical } \\
\text { Immunology } \\
\text { Nánásy-Vass, Melinda; University of Debrecen, University of Debrecen, } \\
\text { Faculty of Medicine, Department of Internal Medicine, Division of Angiology } \\
\text { Veisz, Richárd; University of Debrecen, Faculty of Medicine Department of } \\
\text { Radiology } \\
\text { Bidiga, László; University of Debrecen, Faculty of Medicine Department of } \\
\text { Pathology } \\
\text { Dezső, Balázs; University of Debrecen, Faculty of Medicine Department of } \\
\text { Pathology } \\
\text { Balla, József; University of Debrecen, Faculty of Medicine Division of } \\
\text { Nephrology } \\
\text { Szodoray, Peter; University of Oslo, Department of Immunology Centre for } \\
\text { Immune Regulation } \\
\text { Szekanecz, Zoltán ; University of Debrecen, Faculty of Medicine } \\
\text { Department of Rheumatology } \\
\text { Soltesz, Pal; University of Debrecen, Faculty of Medicine, University of } \\
\text { Debrecen, Faculty of Medicine, Department of Internal Medicine, Division of } \\
\text { Angiology }\end{array}$ \\
\hline Keyword: & SLE, thromboses, catastrophic antiphospholipid syndrome, rituximab \\
\hline Abstract: & $\begin{array}{l}\text { Antiphospholipid syndrome (APS) is characterized by multiple arterial } \\
\text { and/or venous thrombotic events, recurrent fetal losses in the presence of } \\
\text { antiphospholipid antibodies. Catastrophic antiphospholipid syndrome } \\
\text { (CAPS) is a life-threatening, rare subset of APS when the thrombotic } \\
\text { events affect at least } 3 \text { organs, and clinical manifestations develop } \\
\text { simultaneously or within a week. Diagnostically, small vessel occlusions } \\
\text { can be detected by histopathology in the presence of antiphospholipid } \\
\text { antibodies. Our case report describes a } 18 \text { years old man who has been } \\
\text { treated with secondary APS (the underlying disease was Systemic Lupus } \\
\text { Erythematosus) since } 2011 \text {. The clinical findings were dominated by } \\
\text { recurrent deep vein thrombosis, and severe proteinuria caused by lupus }\end{array}$ \\
\hline
\end{tabular}


nephritis, accompanied by mild serological and laboratory findings. The patient was hospitalized in March 2014 because of severe thrombocytopenia and infective diarrhea. At this time the renal functions rapidly deteriorated. Simultaneously left upper extremity paresis was observed; CT showed ischemic lesions in the territory of the middle cerebral artery. Abdominal discomfort and pain occurred. On CT scan ischemic lesions could be seen in the spleen, in the right kidney and in the celiac trunk. Laboratory and serological findings verified the presence of antiphospholipid antibodies and anti-DNA antibodies, anemia and thrombocytopenia. Based on the above mentioned clinical and laboratory findings, the diagnosis of CAPS was established. Anticoagulation, corticosteroid, and plasmapheresis treatment, as well as hemodiafiltration were initiated. Although the thrombotic cascade decelerated following these interventions, we could not see improvement in the renal function. Rituximab treatment was started, leading to significant improvement in the renal function. After 5 weeks of treatment the patient was discharged from the hospital. 


\section{Microthrombotic renal involvement in an SLE patient with concomitant catastrophic antiphospholipid syndrome - The beneficial effect of Rituximab treatment}

Ágnes Diószegi ${ }^{1}$, Tünde Tarr ${ }^{2}$, Melinda Nagy-Vincze ${ }^{2}$, Melinda Nánásy-Vass ${ }^{1}$, Richárd Veisz $^{3}$, László Bidiga ${ }^{4}$, Balázs Dezső ${ }^{4}$, József Balla ${ }^{5}$, Peter Szodoray ${ }^{6}$, Zoltán Szekanecz ${ }^{7}$ and Pál Soltész ${ }^{1}$

${ }^{1}$ University of Debrecen, Faculty of Medicine, Department of Internal Medicine, Division of Angiology, Intensive Care and Therapeutic Apheresis Unit, Debrecen, Hungary

${ }^{2}$ University of Debrecen, Faculty of Medicine, Division of Clinical Immunology, Debrecen, Hungary

${ }^{3}$ University of Debrecen, Faculty of Medicine, Department of Radiology, Debrecen, Hungary

${ }^{4}$ University of Debrecen, Faculty of Medicine, Department of Pathology, Debrecen, Hungary

${ }^{5}$ University of Debrecen, Faculty of Medicine, Division of Nephrology, Debrecen, Hungary

${ }^{6}$ Centre for Immune Regulation, Department of Immunology, University of Oslo; Oslo

University Hospital, Rikshospitalet, Oslo, Norway

${ }^{7}$ University of Debrecen, Faculty of Medicine, Department of Rheumatology, Debrecen, Hungary

\section{Corresponding author: Pál Soltész}

${ }^{1}$ University of Debrecen, Faculty of Medicine, Division of Angiology, Intensive Care and Therapeutic Apheresis Unit, Debrecen, Hungary

4032, Debrecen, Móricz Zsigmond street 22., Hungary

Email: dr.soltersz.pal@gmail.com 
Abstract

Antiphospholipid syndrome (APS) is characterized by multiple arterial and/or venous thrombotic events, recurrent fetal losses in the presence of antiphospholipid antibodies. Catastrophic antiphospholipid syndrome (CAPS) is a life-threatening, rare subset of APS when the thrombotic events affect at least 3 organs, and clinical manifestations develop simultaneously or within a week. Diagnostically, small vessel occlusions can be detected by histopathology in the presence of antiphospholipid antibodies. Our case report describes a 18 years old man who has been treated with secondary APS (the underlying disease was Systemic Lupus Erythematosus) since 2011. The clinical findings were dominated by recurrent deep vein thrombosis, and severe proteinuria caused by lupus nephritis, accompanied by mild serological and laboratory findings. The patient was hospitalized in March 2014 because of severe thrombocytopenia and infective diarrhea. At this time the renal functions rapidly deteriorated. Simultaneously left upper extremity paresis was observed; CT showed ischemic lesions in the territory of the middle cerebral artery. Abdominal discomfort and pain occurred. On CT scan ischemic lesions could be seen in the spleen, in the right kidney and in the celiac trunk. Laboratory and serological findings verified the presence of antiphospholipid antibodies and anti-DNA antibodies, anemia and thrombocytopenia. Based on the above mentioned clinical and laboratory findings, the diagnosis of CAPS was established. Anticoagulation, corticosteroid, and plasmapheresis treatment, as well as hemodiafiltration were initiated. Although the thrombotic cascade decelerated following these interventions, we could not see improvement in the renal function. Rituximab treatment was started, leading to significant improvement in the renal function. After 5 weeks of treatment the patient was discharged from the hospital.

Key words: SLE, thromboses, catastrophic antiphospholipid syndrome, rituximab

Introduction

Antiphospholipid syndrome (APS) is characterized by multiple arterial and/or venous thrombotic events, recurrent fetal losses in the presence of antiphospholipid antibodies. The group of antiphospholipid antibodies consists of various members, such as lupus anticoagulant (LA), anti-cardiolipin antibodies (aCL) and anti-ß2-glycoprotein-I (aß2GPI) antibodies $(1,2)$.

Catastrophic antiphospholipid syndrome (CAPS) is a rare, severe, life-threatening subset of APS, which makes up less than $1 \%$ of all APS cases (3). According to the CAPS Registry, 
$59 \%$ of the patients with CAPS had APS, while $26.9 \%$ suffered from SLE. In most of the cases, various pathogenic factors were verified, mainly infections. Most common infective agents are Salmonella, Escheria coli, Streptococcus, Staphylococcus (4). According to Asherson's study the most frequent infections are respiratory, cutaneous, urinary tract, gastrointestinal infections and sepsis (5).

Others triggering factors can be the discontinuation of anticoagulation therapy, oral contraceptives, or neoplasmas. Recently it was published that besides infection thrombocytopenic state, low level of vitamin D and high level of ferritin also can induce CAPS (4). The pathomechanism of CAPS in not fully understood, while a series of pathogenic effects has been implicated. Anti-phospholipid antibodies trigger a prothrombotic state, while inflammation ultimately leads to clot formation. Uncontrolled activation of the complement system also can initiate and amplify of pathological features of CAPS. Another mechanism is denoted as 'cytokine storm' which is caused by the release of proinflammatoric cytokines (IL-1, IL-5, IL-6, IFN- $\alpha$, TNF- $\alpha$ ) from affected tissues during thrombotic events mainly affecting the microvessels (4). The cytokines activate the innate immune system via TLR-4 production which can be induced by lipopolysacharides derived from bacteria (6).

On the other hand, fibrinolysis is decreased, because of the high levels of plasminogen activator inhibitor-1 (PAI-1). Asherson published the first diagnostic criteria for CAPS (7), later which was revised and is in use since then (8). According to the CAPS Registry the most frequently affected organs are the kidneys, lungs, brain, heart and the skin, while less frequently affected organs are the peripheral vessels, intestine, spleen, pancreas, retina and the bone marrow. CAPS has a high mortality; before 2000 the mortality rate was $50 \%$ despite treatment (9). The therapy has three main aims. If there is an identifiable triggering factor, the treatment of that condition improves CAPS symptoms. In case of infection, antibiotics or antiviral therapy should be initiated. The other crucial point is to decelerate the thrombotic cascade and to suppress the cytokine storm, which leads to Systemic Inflammatory Response Syndrome (SIRS). The first line therapy consists of anticoagulation and corticosteroid treatment. Plasma exchange and/or intravenous immunoglobulin can have favorable effects on the disease course. If CAPS is associated with autoimmune disease, mainly SLE immunesupression could be considered. Cyclophosphamid is most frequently used in these cases. In therapy refractory or relapsing cases new therapeutic options need to be considered.

Rituximab is a monoclonal antibody against CD20, which is expressed on B cells. Rituximab downregulates B-cell receptors and induce B-cell apoptosis. B-cell depletion may also reduce 
the production of proinflammatory cytokines which leads to the modulation of SIRS. Rituximab has been approved in non- Hodgkin lymphoma, rheumatoid arthritis, SLE. There are ongoing clinical trials with rituximab in therapy resistant non-criteria manifestations, such as thrombocytopenia, ulcers, hemolytic anemia of APS. According to the RITAPS trial rituximab was safe in APS patients, however rituximab has not induced changes in the antiphospholipid antibody profile, but it was effective in non-criteria manifestations of APS.

According to data from the CAPS registry, rituximab reduced mortality to $20 \%$. Kidney involvement was the most frequent, followed by cardiac and cerebral involvement. Rituximab was second line therapy in $60 \%$ of the cases because of poor response to first line therapy, recurrent CAPS, therapy resistant thrombocytopenia or new thrombotic event $(11,12)$.

Over the last 15 years 4 patients have been treated with CAPS in our Department. The first cancer associated CAPS was published in 2000 (13). The 45 years old woman was admitted to the ICU with neurological symptoms. The patient did not have APS or systemic autoimmune disease in her medical history. The clinical situation was dominated by rapid onset of multiple thrombotic events which affected the brain, spleen, kidneys, lungs and the mitral valve with the presence of anti-cardiolipin and anti-beta2GPI antibodies, which fulfilled the diagnostic criteria for CAPS. The patient died in multi-organ failure after 2 weeks despite anticoagulation and corticosteroid treatment. The predisposing factor was adenocarcinoma of the stomach, but it was revealed during autopsy. Our second case of CAPS was also associated with malignancy. The 62 years old woman was diagnosed with cytokeratin-7 positive endometrial cancer 1 year before CAPS was diagnosed. She was admitted to the hospital with pulmonary embolism. Anticoagulation therapy started. Pericardial and pleural effusion were revealed on echocardiography. Vascular ultrasound demonstrated the presence of thromboses in the internal jugular vein and inferior cava vein. Anticardiolipin IgG antibody positivity was tested. Her condition despite therapy deteriorated, the neurological status progressed to coma, intubation and mechanical ventilation was necessitated. 4 days after admission she died. Autopsy revealed multiple thromboses of the pelvic vein, portal and hepatic veins. Microtrombi was observed in the spleen. Immunehistochemical staining of the lungs showed cytokeratin-7 positivity, which indicated the metastasis of the previous endometrial cancer. The clinical picture supported the diagnose of CAPS which was verified by autopsy and histopathology (14). 
In our third case CAPS was associated with SLE. SLE showed moderate and mild clinical and serological activity including antiphospholipid antibody positivity in low concentration (anti-B2-glycoprotein IgG and anti-prothrombin), but no thrombotic clinical symptoms have been observed. In December 2012 she was hospitalized due to pulmonary embolism preceded by a lower respiratory tract infection. Anticoagulation with therapeutic dose low molecular weight heparin (LMWH) have been started. Despite of anticoagulation, neurological symptoms appeared. Head CT showed cerebrovascular thromboses. Her condition rapidly deteriorated, multi-organ failure developed. Multiple infarctions in the spleen and kidneys could be detected in abdominal CT. Renal biopsy showed occlusive vasculopathy in the small vessels. Biopsy also proved chronic active mesangioproliferative glomerulonephritis with mesangial immune deposits. Lab tests indicated APA positivity in high titer. Overall, the clinical picture fulfilled the criteria of catasrophic antiphospholipid syndrome. In this case, CAPS was triggered by systemic autoimmune disease and infection. The anticoagulation and corticosteroid treatment was supplemented by high volume plasma exchange which was synchronized by intravenous immunoglobuline. Due to the immunmodulatory treatment the patient recovered. Since then no thrombotic events have been occurred, and clinically, the SLE symptoms are in remission.

\section{Methods}

The 19 years old, Caucasian, male patient was treated with severe thrombocytopenia, indirect Coombs positivity and suffusions. Intravenous immunoglobulin was administered, thrombocyte level was normalized Immunological tests were performed which showed antibeta2GPI, anti-cardiolipin, and anti-phosphetidilserine antibody positivity, but there was no thrombotic event at that time. In September 2010 he was diagnosed with deep vein thrombosis of the right femoral vein, as well as the communal and external iliacal vein. Thrombophilic tests (FII20210A, AT-III, PS and PC activity, FV Leiden, APS screening) were performed at the time; LA, aCL and aß2GPI antibodies were identified again. Oral anticoagulant therapy with vitamin $\mathrm{K}$ antagonist was started In 2011 he was diagnosed with SLE based on photosensitivity, lupus erythema and the presence of anti-nuclear, anti-SSAanti-SSB antibody, anti-CL antibodies and hypocomplementaemia, as well as positive LA. The diagnosis of secondary APS was also established based on the clinical picture and laboratory findings. The maintenance therapy was corticosteroid, hydroxycloroquine and 
anticoagulation. During the next 2 years he has been in remission. He had mild symptoms of arthralgia, skin involvrment and mild upper airway tract infections.

During the autumn of 2013 he was hospitalized several times because of high sedimentation rate, massive proteinuria (10 g/day), massive edema on the legs, increasing levels of carbamide, creatinine, worsening anemia and thrombocytopenia. After pulse corticosteroid treatment $(3 \times 1000 \mathrm{mg})$, the platelet counts increased. Due to deteriorating renal function and massive proteinuria, as the sign of the activation of lupus, mycophenolate mofetil treatment was initiated. In January 2013 he was hospitalized because of recurrent deep vein thrombosis in his right superficial femoral vein. Low molecular weight heparin therapy was administered. The therapy was modified due to recurrent thromboses and activation of SLE: Cyclophosphamid was initiated with good therapeutic response; the proteinuria diminished and the renal functions slightly improved.

In March 2014 he was hospitalized for the next cycle of cyclophosphamide therapy. However, he could not get the next cycle of cyclosphamide because the platelet count was under 150 G/L. In the physical status, swollen lilac lips, epigastric pain, edema of the lower right extremity and diarrhea could be outlined. The lab test showed high CRP levels $(90.51 \mathrm{mg} / \mathrm{mL}$; normal range: $<4.6 \mathrm{mg} / \mathrm{L})$ leukocytosis $(12.72 \mathrm{G} / \mathrm{L}$; normal range:4.5-10.8 $\mathrm{G} / \mathrm{L})$, thromobocytopenia (31 G/L; normal range: 150-400 G/L) and anemia (Hgb: $123 \mathrm{~g} / \mathrm{L}$; normal range: $130-165 \mathrm{~g} / \mathrm{L})$, increased carbamide $(23.3 \mathrm{mmol} / \mathrm{L}$; normal range: $3.6-6 \mathrm{mmol} / \mathrm{L})$ and creatinine $(464 \mu \mathrm{mol} / \mathrm{L}$; normal range: $44-97 \mu \mathrm{mol} / \mathrm{L})$ levels, hypoproteinemia $(52 \mathrm{~g} / \mathrm{L}$; normal range: $>60 \mathrm{~g} / \mathrm{L})$ and high fibrinogen levels $(9.29 \mathrm{~g} / \mathrm{L}$; normal range: $1,5-4 \mathrm{~g} / \mathrm{L})$. The clinical picture and the lab tests confirmed the diagnosis of gastrointestinal infection. Metronidazole was initiated. In the next few days the gastrointestinal symptoms diminished, however the renal failure, anemia, thrombocytopenia progressed and in the quantitative blood smear fragmentocytes were detected. Subsequently paresis of the left upper extremity appeared. Skull CT showed ischemic lesion at the territory of the right medial cerebral artery (Figure 1A). Due to exceeding gastrointestinal pain, abdominal CT was performed, which detected thrombus in the lumen of the celiac trunk (Figure 1B), infarction in the spleen (Figure 1C) and in the right kidney (Figure 1D). Lab tests revealed anti-double stranded DNA, anti-beta-GPI positivity. As a differential diagnosis thrombotic trombocytopenic purpura/hemolytic uremic syndrome (TTP/HUS) was established, however it was ruled out by negative results on ADAMTS 13 and anti-Factor $\mathrm{H}$ antibody assessment. Renal biopsy was performed which revealed thrombotic nephropathy. Immunohistochemistry showed 
microthrombi which occluded the glomerular capillaries. The biopsy specimen revealed SLEassociated chronic glomerular damage exhibiting scattered thrombotic occlusions of the capillary tufts reflecting CAPS (Figure 2.). Immunofluorescent staining for fibrin confirmed that the glomerular capillary tufts' occlusions (long arrows) and the interstitial vascular obstructions (thick arrows) correspond to fibrin-rich thrombi (green fluorescence), suggestive of renal manifestation of CAPS (original magnification: 40x; nuclear counterstain is with the blue fluorescent DAPI). Both confirm the multiple intraglomerular fibrin- thrombi (arrows) which in part are found along the intimal-subendothelial region of the capillary tuft (image $\mathrm{C}$, arrows). Electron microscopy (EM) exhibited glomeruli with basement membrane (BM) thickening and endothelial-subendothelial damages associated with CAPS (Figure 3). Moreover, higher magnification (10000x) identifies splitting and dissection of the endothelial cells from the BM in association with electron-lucent subendothelial (SE) amorphous material deposition admixed with tissue debris. Finally, double immunofluorescent labeling of the biopsy specimens identified the presence of the membrane-attack complex of the complement cascade that showed unambiguous evidence of endothelial and subendothelial deposition and insudation of C9 that is predominantly manifested along the glomerular capillary tufts and in part within the vasculature of the interstitial spaces (Figure 4). Further stainings for T- and Bcells showed the immune-inflammatory cellular composition of the SLE-associated CAPS in this case, demonstrating that both the T-cells and the B-lymphocytes could be considered to have roles in the pathomechanism of the vascular damage resulting in thrombotic occlusions (Figure 4).

\section{Results}

Based on the clinical features histology and laboratorial findings, the diagnosis of gastrointestinal infection induced CAPS on the grounds of SLE, perpetuated by the complement cascade and T-/B-lymphocytes was established.

The primary goal of the therapeutic plan was to decelerate the thrombotic cascade and to suppress inflammatory cytokines. LMWH treatment was continued but in lower dosage $(2 \times 0.2 \mathrm{ml})$ in order to avoid bleeding in the ischemic lesion of the territory at the right medial cerebral artery. The dose of corticosteroid was increased to $80 \mathrm{mg}$ per day. Plasmapheresis treatment $(40 \mathrm{ml} / \mathrm{kg})$ was performed 6 times. Hemodiafiltration treatment was performed 3 times because of the acute renal failure and increasing levels of carbamide and creatinine. 
After 6 plasmapheresis cycles, along with the corticosteroid and LMWH treatment, the paresis slightly resolved, and the hematological parameters began to normalize. However, no significant improvement could be achieved in the renal function. The therapeutic regime was therefore supplemented with Rituximab. $1000 \mathrm{mg}$ Rituximab was given two times and the treatment led to the significant improvement of the acute renal failure. Depicts the efficacy of therapy on renal failure. After 5 weeks the patient was discharged from the hospital with normal laboratory parameters and good general health condition. After 1 year the patient had a grand mal seizure at home. He was admitted to the local hospital where skull CT was performed. The CT scan could not verify new ischemic lesion or bleeding. He was transferred to our intensive care unit. Upon arrival he was cardio-respiratory compensated. Lab tests revealed normal Hgb, platelet, leucocytes levels. The CRP, PCT was in normal range, which ruled out any underlying infective cause. Antiphospholipid antibodies were negative, as well. On the first day grand mal seizure appeared again, which was followed by Todd paresis. To ease the seizure $4 \mathrm{mg}$ midazolam and $10 \mathrm{mg}$ diazepam was given intravenously. Possibly the seizures were caused by the central nervous system involvement of SLE. To rule out cerebral vasculitis skull MR was performed, which only showed the signs of previous ischemic lesions. Vasculitis, bleeding or new thrombotic event could not be detected on MR. EEG was also performed which detected epileptic activity. The therapy was supplemented by carbamazepine. During hospitalization epileptic seizures were not repeated. Neither thrombotic events, nor kidney failure appeared. The kidney function was compensated. The carbamide level was $10.4 \mathrm{mmol} / \mathrm{l}$, while creatinine was $158 \mathrm{umol} / \mathrm{l}$. During urine analysis proteinuria could not be detected. He was discharged from the hospital after 1 week in a good overall condition. Since then he is free of seizures, he takes $400 \mathrm{mg}$ carbamazepine daily. SLE is in remission with the maintenance therapy of corticosteroid, hydroxycloroquine and anticoagulation.

\section{Discussion}

This complex case shows multi-organ thrombotic escalation. We believe that in this patient the concomitant infection initiated pathological processes, such as binding of antiphospholipid antibodies to the endothelium, followed by complement activation. The accelerated complement activation, therefore, as a second hit led to the development of catastrophic thrombotic manifestations. We also showed the intrarenal accumulation of lymphocytes, both $\mathrm{T}$ - and B-cells, assuming that these cells also play a role in the 
development of renal pathology. These findings may explain the favorable effect of Rituximab treatment on renal function, which is beneficial for decelerating lupus-associated humoral autoimmune processes, as well as the CAPS-associated pro-thrombotic antiphospholipid antibody production. In our case immunohistochemistry reinforced the double-hit theory in CAPS development, identifying the presence of complements, as well as B-cells intrarenally, giving rise to the beneficial effects of using plasmapheresis and Rituximab in the disease management.

In the standard management of CAPS anticoagulation, corticosteroid, plasmapheresis and immunemodulatory treatment is utilized, as well as the targeted therapy of other etiological factors (e.g. infections) is pivotal. However, in certain cases, the modulation or depletion of B cells can be necessary, as the source of autoantibodies and pro-inflammatory cytokines (7). According to the CAPS Registry only 20 cases treated with Rituximab have been reported so far (8). In most cases it was a second line treatment, based on poor therapeutic response to first line therapy. In 4 cases Rituximab was the first line therapy because of associated lymphoma or very severe, other life-threatening medical conditions. Among patients treated with Rituximab, the mortality decreased to $20 \%$; among the treated patients 4 out of 20 died (8).

\section{Conclusion}

We believe that in our patient, the identification of intrarenal complement activation and T/B lymphocytic infiltration along with diffuse microthrombus formation aid in understanding the pathomechanism of lupus-associated CAPS triggered by infection. Based on histological evidence a combination therapy, including Rituximab treatment was used successfully, due to non-responding acute renal failure. After rituximab treatment the antiphospholipid antibody profile has been changed. According to data from the CAPS registry the effect of rituximab on antiphospholipid antibodies was controversial. In most cases the antibody profile did not change after rituximab treatment. Overall we suggest that in these patients B-cell depletion or plasma cell modulation can be considered and can be beneficial.

Conflict of interests: There is no conflict of interests. 


\section{References}

1. Cervera R, ReverterJC, Khamashta MA, Antiphospholipid syndrome in systemic autoimmune diseases, Amsterdam:Elsevier; 2009)

2. Hughes GR. Thrombosis, abortion, cerebral disease, and the lupus anticoagulant. Br Med J (Clin Res Ed). 1983 Oct 15;287(6399):1088-9.

3. Asherson RA, The catastrophic antiphospholipid syndrome. J Rheumatol.1992 Apr;19(4):508-12.)

4. Cervera R, Rodríguez-Pintó I, Colafrancesco S, Conti F, Valesini G, Rosário C, AgmonLevin N, Shoenfeld Y, Ferrão C, Faria R, Vasconcelos C, Signorelli F, Espinosa G 14th International Congress on Antiphospholipid Antibodies Task Force Report on Catastrophic Antiphospholipid Syndrome. Autoimmun Rev. 2014 Jul;13(7):699-707. doi: 10.1016/j.autrev.2014.03.002. Epub 2014 Mar 20.)

5. Asherson RA, Cervera R, Piette JC, Shoenfeld Y, Espinosa G, Petri MA, et al. Catastrophic antiphospholipid syndrome: clues to the pathogenesis from a series of 80 patients. Medicine (Baltimore) Nov 2001;80(6):355-77.

6. Autoimmun Rev. 2015 Nov;14(11):1066-71. doi: 10.1016/j.autrev.2015.07.009. Epub 2015 Jul 22. The role of infectious diseases in the catastrophic antiphospholipid syndrome. GarciaCarrasco M1, Mendoza-Pinto C1, Macias-Diaz S2, Vazquez de Lara F3, Etchegaray-Morales I2, Galvez-Romero JL4, Mendez-Martinez S5, Cervera R6.)

7. Asherson RA1, Espinosa G, Cervera R, Font J, Reverter JC. Catastrophic antiphospholipid syndrome: proposed guidelines for diagnosis and treatment. J Clin Rheumatol. 2002 Jun;8(3):157-65

8. Asherson RA1, Cervera R, de Groot PG, Erkan D, Boffa MC, Piette JC, Khamashta MA, Shoenfeld Y; Catastrophic Antiphospholipid Syndrome Registry Project Group Catastrophic antiphospholipid syndrome: international consensus statement on classification criteria and treatment guidelines. (11, Lupus. 2003;12(7):530-4.

9. Espinosa G1, Berman H, Cervera R). Management of refractory cases of catastrophic antiphospholipid syndrome. Autoimmun Rev. 2011 Sep;10(11):664-8. doi: 10.1016/j.autrev.2011.04.031. Epub 2011 May 1. 
10. Erkan D1, Vega J, Ramón G, Kozora E, Lockshin MD.) A pilot open-label phase II trial of rituximab for non-criteria manifestations of antiphospholipid syndrome. Arthritis Rheum. 2013 Feb;65(2):464-71. doi: 10.1002/art.37759.

11. Bakshi J, Stevens R. Rituximab therapy for recurrent thromboembolic disease in antiphospholipid syndrome. Lupus. 2013 Jul;22(8):865-7. doi: 10.1177/0961203313491023. Epub 2013 Jun 5)

12. Berman H1, Rodríguez-Pintó I, Cervera R, Morel N, Costedoat-Chalumeau N, Erkan D, Shoenfeld Y, Espinosa G; Catastrophic Antiphospholipid Syndrome (CAPS) Registry Project Group (European Forum on Antiphospholipid Antibodies). Rituximab use in the catastrophic antiphospholipid syndrome: descriptive analysis of the CAPS registry patients receiving rituximab. Autoimmun Rev. 2013 Sep;12(11):1085-90. doi: 10.1016/j.autrev.2013.05.004. Epub 2013 Jun 15.)

13. Soltész $P^{1}$, Szekanecz Z, Végh J, Lakos G, Tóth L, Szakáll S, Veres K, Szegedi G. Catastrophic antiphospholipid syndrome in cancer. Haematologia (Budap). 2000;30(4):30311.

14. Soltész $\mathrm{P}^{1}$, Veres K, Szomják E, Kerekes G, Dér H, Sándor Z, Dezso B, Dévényi K, Szekanecz Z. Catastrophic antiphospholipid syndrome (Asherson's syndrome) associated with cytokeratin 7-positive endometrial cancer. Isr Med Assoc J. 2007 Dec;9(12):891-3. 


\section{Legend to figures}

Figure 1. CT image of ischaemic laesion (A), thrombus in the coliac trunk (B), spleen infarction (C) and kidney infarction (D)

\section{Figure 2.}

Hematoxylin-eosin (HE) stained glomerular biopsy specimen (A), Immunofluorescent staining for fibrin (B), Trichrome special stainings of multiple intraglomerular fibrin- thrombi (C-D)

\section{Figure 3.}

Electron microscopy (EM) specimens of glomeruli. Screening magnification (3000x) (A), Higher magnification (10000x) (B)

\section{Figure 4.}

Double immunofluorescent labeling of the membrane-attack complex (A) and the pan-T-cell combined with the pan-B-cell marker to show the immune-inflammatory cellular composition (B) 

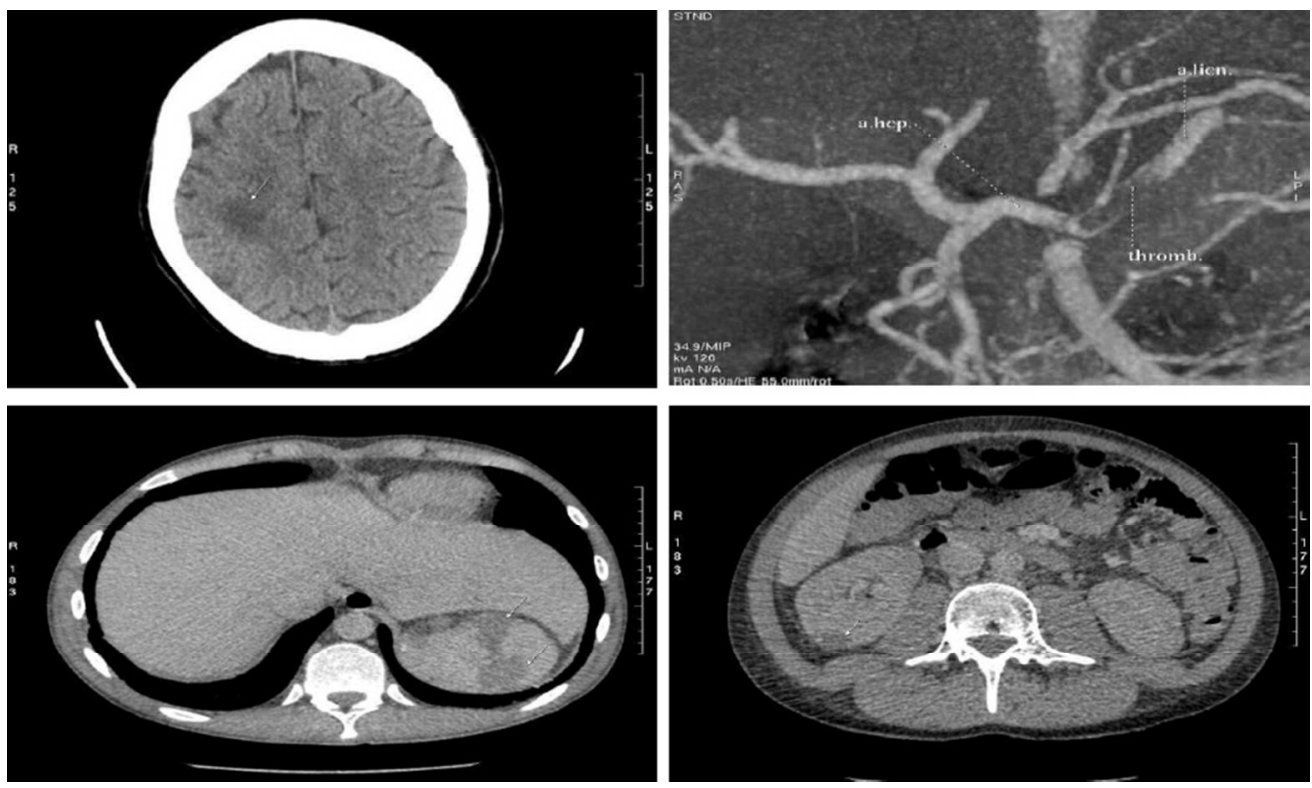

Figure 1. CT image of ischaemic laesion (A), thrombus in the coliac trunk (B), spleen infarction (C) and kidney infarction (D)

$172 \times 102 \mathrm{~mm}(144 \times 144$ DPI $)$ 


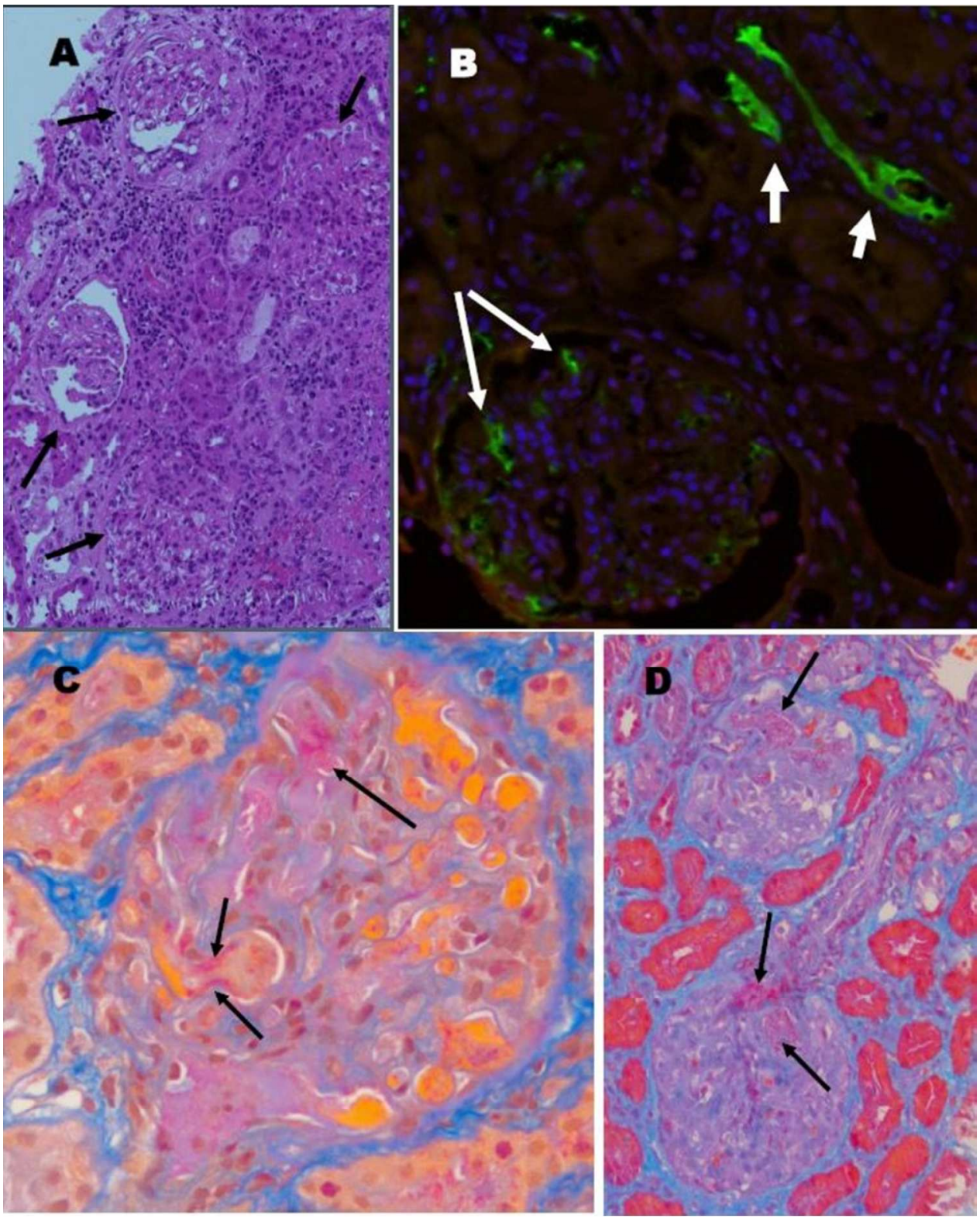

Figure 2.

Hematoxylin-eosin (HE) stained glomerular biopsy specimen (A), Immunofluorescent staining for fibrin (B), Trichrome special stainings of multiple intraglomerular fibrin- thrombi (C-D) 


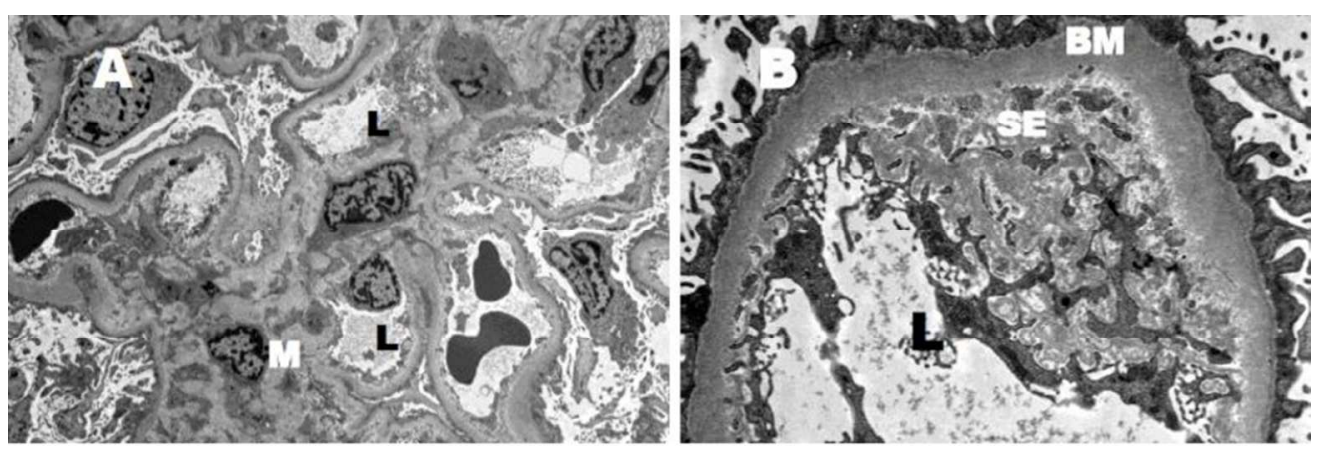

Figure 3.

Electron microscopy (EM) specimens of glomeruli. Screening magnification (3000x) (A), Higher magnification (10000x) (B)

$159 \times 52 \mathrm{~mm}(144 \times 144 \mathrm{DPI})$ 


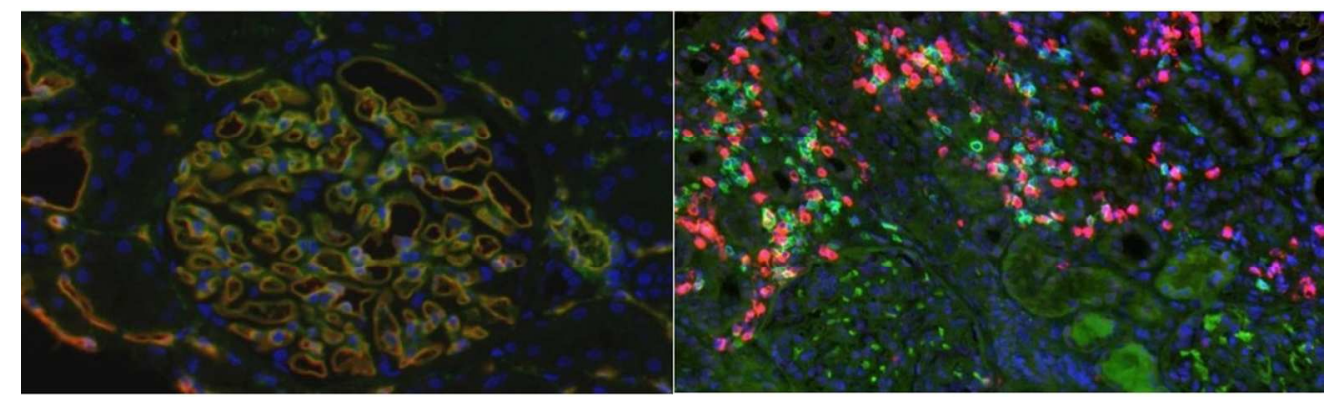

Figure 4.

Double immunofluorescent labeling of the membrane-attack complex $(A)$ and the pan-T-cell combined with the pan-B-cell marker to show the immune-inflammatory cellular composition (B)

$192 \times 57 \mathrm{~mm}(144 \times 144 \mathrm{DPI})$ 